\title{
Estudo estatistico sobre a frequencia de parazitos intestinais nas crianças do Rio de Janeiro.
}

PELO

Dr. Gomoe do Iraria.

\section{Statistische Studien über die Häufigkeit der Darmparasiten bei den Kindern von Rio de Janeiro.}

\author{
VON
}

Dr. Gomen de Iaria.

No prezente trabalho damos uma contribuição para a estatistica dos parazitos intestinaes, bazeada no abundante material fornecido pelos numerozos doentes que frequentam a Policlinica de Crianças.

Logo que assumimos a direção do Laboratorio dessa Policlinica ocorreu-nos estudar a frequencia de parazitos intestinaes nas crianças do Rio de Janeiro, considerada sob varios pontos de vista como sejam sexo e idade dos hospedeiros, predominancia de certas especies, frequencia das associações, etc.

Podemos agora dar varias informações sobre este assunto, bazeando os nossos rezultados sobre o exame de 1238 individuos.

Antes de entrar em assunto queremos deixar rejistados aqui os nossos agradecimentos ao Dr. Fernandes Figueira, diretor da Policlinica, pelo interesse que tomou pelas nossas observações. Ao assistente do Laboratorio Snr. J. J. Maciel temos a agradecer os multiplos exames que praticou
In nachfolgender Arbeit gebe ich einen Beitrag zur Statistik der Darmparasiten auf Grund des zahlreichen Krankenmateriales der hiesigen Kinderpoliklinik.

Als ich die Leitung des Laboratoriums dieser Poliklinik übernahm, kam mir sofort der Gedank, die Häufigkeit der Darmparasiten bei den Kindern von Rio de Janeiro zu studieren und zwar unter Berücksichtigung von Geschlecht und Alter der Wirte, Vorwiegen gewisser Arten, Häufigkeit der Assoziationen und so weiter. Ich kann jetzt auf Grund der Untersuchung von $123^{8}$ Kindern über diese Fragen berichten.

Bevor ich auf mein Thema eingehe möchte ich noch dem Direktor der Poliklinik, Herrn Dr. Fernandes FIGUEIRA für das Interesse, welches er meinen Beobachtungen schenkte, meinen besten Dank aussprechen. Dem Assistenten des Laboratoriums Herrn J. J. MACrEL, verdanke ich zahlreiche Untersuchungen, welche er vornahm, sowie den Herren AL-

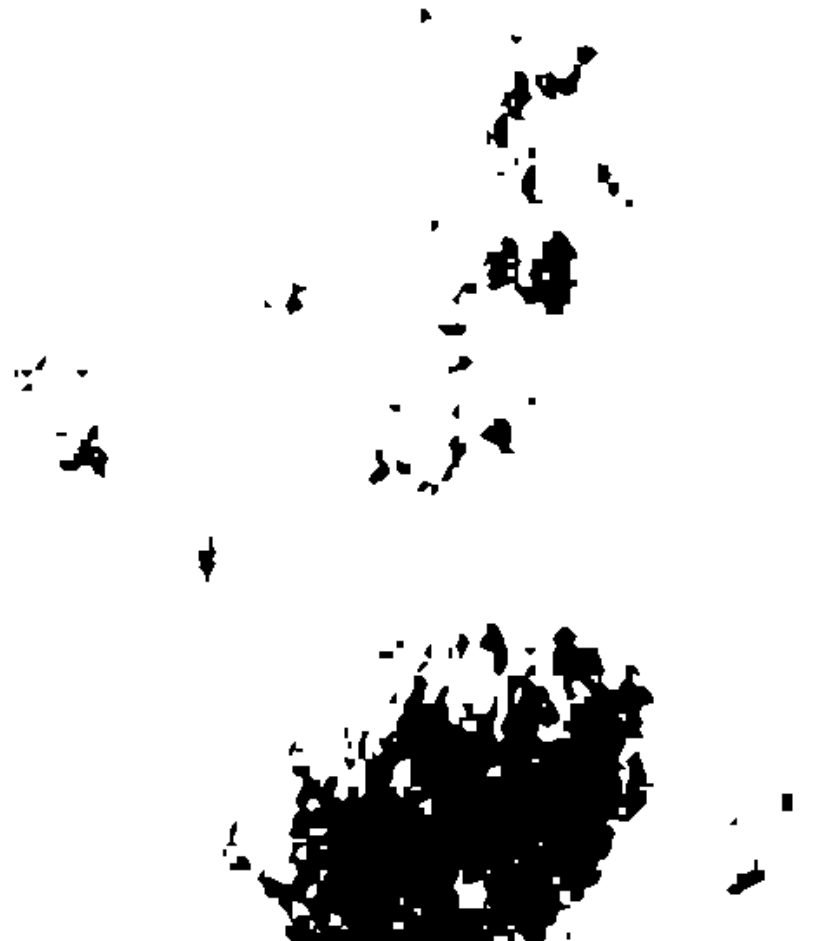


e aos Snrs. Alfredo Neves e Virginio Campello o auxilio que nos prestaram na organização dos dados estatisticos.

Material e tecnica: Em todos os exames para pesquiza de ovos empregámos sempre preparações a fresco, seguindo a tecnica habitual de diluir um pequeno fragmento das fezes compactas com uma gota de agua. As fezes diarreicas muito fluidas eram em geral centrifugadas e o depozito utilizado para preparações entre lamina e laminulas. Esta pratica se nos afigurou muito util embora desprezada por alguns autores como desnecessaria. De cada cazo faziamos 3 a 4 preparações empregando em uma lamina 2 laminulas de $18 \times 18 \mathrm{~mm}$.

Logo no inicio destas observações tivemos noticia do metodo proposto por TELLEMANN, experimentando-o varias vezes. Trabalhando sempre nas mesmas condições do autor tivemos bons rezultados, achámos, porém, objeções bastante serias ás suas vantajens. Incontestavel é que por tal processos se consegue aumentar o numero de ovos no campo microscopico e mesmo fazel-os aparecer quando o simples exame direto dava rezultado negativo. $\mathrm{O}$ acido cloridrico concentrado, que se emprega, exerce ação bastante prejudicial sobre certos ovos, principalmente sobre os ovos de Ascaris lumbricoides, sendo a casca interior fortemente atacada, descorada e mesmo em parte dissolvida, mormente nas formas carateristicas dos ovos não fecundados, bem descritas por Lutz (1888) e MiURA e Nishiuchi (I902). São sobretudo estas formas que as tornam irreconheciveis para quem não tenha larga pratica destas pesquizas.

No nosso meio, em que a ocorrencia das formas não fecundadas em crianças de baixa idade é bastante frequente, oferecia este metodo as desvantagens mencionadas. fredo Neves und Virginio Campello ihre Unterstuitzung bei der Anfstellung der statistischen Berechnungen.

Material und Technik. - Bei allen Untersuchungen auf Eier machte ich frische Präparate, indem nach der gewöhnlichen Technik em kleines Stück fester Fäces mit einem Tropfen Wasser verdünnt wurde. Sehr flüssige Dejektionen wurden gewöhnlich zentrifugiert und der Niederschlag $\mathrm{zu}$ frischen Präparaten verwandt. Dieses Verfahren erwies sich mir als sehr brauchbar, trotzdem es von einigen Autoren als unnütz verworfen wird. Von jedem Falle machte ich drei bis vier Präparate, indem ich je zwei Deckgläser von $18 \mathrm{~mm}$. im Quadrat zu einem Objektträger gebrauchte.

Gleich zu Beginn dieser Beobachtungen erhielt ich Kenntnis von einem von TELLEMANN vorgeschlagenen Verfahren, welches ich dann mehrmals versuchte. Indem ich unter denselben Bedingungen arbeitete, wie der Autor erhielt ich gute Resultate, fand jedoch auch den Vorzügen gegenüber ziemlich gewichtige Nachteile. Es ist unbestreitbar, dass man bei diesem Verfahren eine Vermehrung der Eier im Gesichtsfelde erzielt; gelegentlich findet man sie auch dann, wenn die gewöhnliche Untersuchung ein negatives Resultat ergab. Dagegen übt die konzentrierte Salzsäure, welche dabei angewendet wird, auf manche Eier einen ziemlich nachteiligen Einfluss aus, besonders auf diejenigen von Ascaris lumbricoides, indem die äussere Schale stark angegriffen, entfärbt und teilweise aufgelöst wurde, besonders bei den charakteristischen Formen der unbefruchteten Eier, wie sie 1888 von LUTz und 1902 von MiURa und Nishiuchi genau beschrieben wurden. Gerade diese Formen sind so für denjenigen, der in solchen Untersuchungen nicht sehr bewandert ist, schwer zu erkennen. Hier zu Lande, wo das Vorkommen derselben bei jungen Kindern ziemlich häufig ist, war dies ein unzweifelhafter Nachteil. Wenn das Reagens auch auf andere Eier keine so deutliche Einwirkung zeigt, fand ich doch, 
Comquanto a ação do reativo sobre outros ovos não seja tão pronunciada como no cazo do Ascaris, porem notamos ainda que os de ancilostomos são ás vezes bastante deformados. Ovos de Trichocephalus, Oxyurus e Taenia saginata tornam-se apenas mais claros pela ação descorante do acido. Um ponto importante de que queremos falar é da ação que o liquido de Tellemann exerce sobre as larvas de nematoideos que aparecem nas fezes, como as de Ancylostomum e as de Strongyloides stercoralis. Estas larvas são instantaneamente mortas e deformadas, perdendo as carateristicas importantes para o diagnostico diferencial, tornado facil pelas cuidadozas investigações de LEICHTENSTERN; mas sendo como elle bazeado em dados de anatomia fina, as alterações produzidas impedem qualquer tentativa de diferenciação.

Tambem experimentámos um processo aconselhado por TORIOJI (I9IO) em substituição ao de TeliemanN. Torioji substitue o acido cloridrico por uma solução de sulfato de cobre amoniacal e acha indiferente o emprego de eter sulfurico ou de eter de petroleo. Este metodo não parece facilitar em nada a pesquiza e pomos seriamente em duvida a informação do autor de que os ovos mantêm até a vitalidade.

Pouco antes da terminação deste trabalho apareceu um artigo de MaURICE HaLL (I9II) em que faz um estudo comparativo dos metodos de exame das fezes para pesquiza de ovos de parazitos. Aqui são passados em revista mais alguns novos metodos que não foram experimentados por nós.

Em todas as nossas pesquizas uzavamos fazer sempre uma inspeção macroscopica das fezes, que näo deve nunca ser dispensada, não sendo raro encontrar vermes adultos como sejam Oxyurus, cujos ovos raramente são encontrados no exame microscopico, e proglotides de Taenia que impõem imediatamente um diagnostico. dass auch diejenigen von Ancylostomum manchmal ziemlich deformiert werden. Die Eier von Trichocephalus, Oxyurus und Taenia saginata werden durch die Einwirkung der Säure nur heller. Ein wichtiger Umstand, den ich erwähnen möchte, liegt in der Einwirkung, welche die von Tellemann empfohlene Flüssigkeit auf die in den Fäces enthaltenen Nematodenlarven, wie Ancylostomum und Strongyloides stercoralis, ausübt. Dieselben werden augenblicklich getötet und so verändert, dass sie die für die Unterscheidung wichtigen Kennzeichen verlieren. Diese Unterscheidung ist in Folge der genauen Studien von LEICHTENSTERN nicht schwierig, basiert sich aber auf feine anatomische Détails, so dass die hervorgebrachten Veränderungen jeden Versuch einer Bestimmung erfolglos machen.

Ich versuchte auch ein von ForIojI (igio) an Stelle des von Tellemann empfohlenen Verfahrens. Er ersetzte die Salzsäure durch eine Lösung von ammoniakalischem Kupfersulfat und hält es für gleichgültig, ob man Schwefel-oder Petroleumether gebrauche. Diese Methode scheint mir die Untersuchung in keiner Weise zu erleichtern und ich hege auch ernstlichen Zweifel an der Richtigkeit der Angabe, dass die Eier selbst ihre Lebenskraft bewahren.

Kurz vor Vollendung dieser Arbeit erschien auch ein Artikel von MAURICE Hall (I9II), der ein vergleichendes Studium der Methoden für die Untersuchung der Fäces auf Parasiteneier enthält. Hier werden auch einige neue Methoden besprochen, welche ich noch nicht ausprobiert habe.

Bei allen meinen Untersuchungen pflegte ich stets eine makroskopische Untersuchung der Fäces vorzunehmen, welche niemals unterbleiben sollte, da man nicht selten ausgewachsene Würmer findet, wie z. B. den Oxyurus, dessen Eier nur selten bei der mikroskopischen Untersuchung gefunden werden, oder Bandwurmglieder, welche die direkte Diagnose gestatten. 
Rezultados - Submetemos a exame as fezes de 1238 crianças de todas as idades até 12 anos. Destas 662 mostraram a prezença de parazitos intestinais ou $53,4 \%$.

Por ordem de frequencia damos os rezultados em relação a especie de parazitos encontrados :

Trichocephalus trichiurus . 446 vezes ou $67,0 \%$ Ascaris lumbricoides . . 432 » $\$ 65,2 \%$ Ancylostomum duod. ì

Necator amer. . . . . I20 》 $16,7 \%$ Strongyloides stercoralis: 58 \% $8,7 \%$ Taenia sanginata . . . 22 \ Oxyurus vermicularis . . I \ $1,6 \%$

Em relação a frequencia das associações parazitarias observadas, aprezentamos os dados seguintes :

Ascaris e Trichocephalus ..$~$
Ascaris, Trichocephalus e Ancylos-

Infestações determinadas por um só parazito foram encontradas nas seguintes proporções :

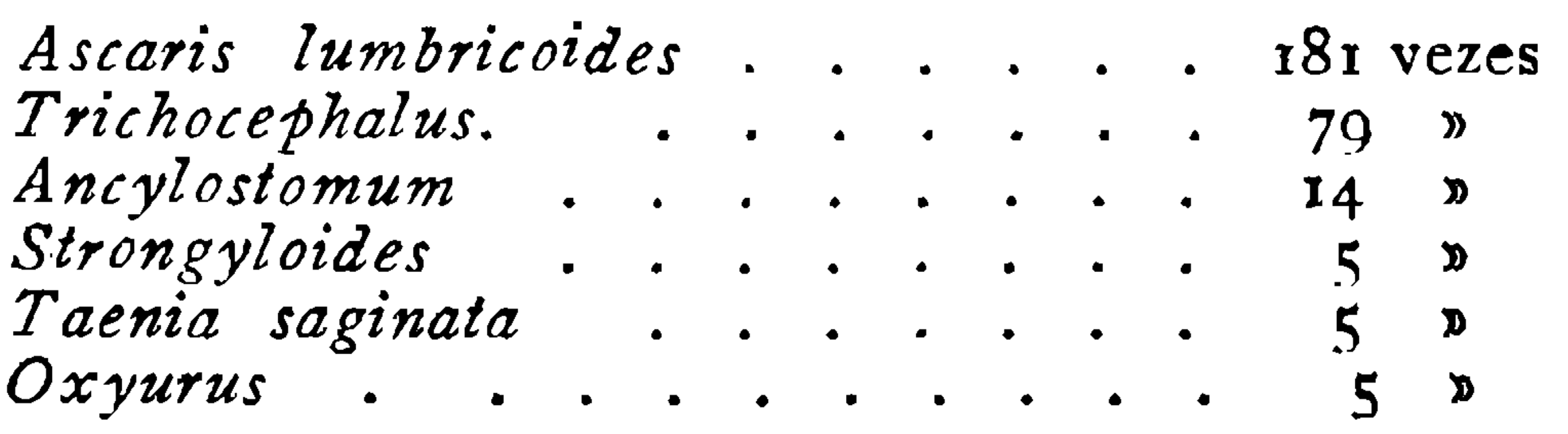

Para mostrar a frequencia de parazitos conforme as idades organizámos o seguinte quadro, mostrando as percentajens para cada sexo, separadamente:
Resultate: Es wurden Fäzes von I $z 38$ Kindern untersucht, welche alle Altersstufen bis zu I 2 Jahren repräsentierten.

Von diesen zeigten 662 oder $53,4 \%$ die Gegenwart von Parasiten. Ich gebe hier die Verhältniszahlen der einzelnen beobachteten Parasiten in der Reihenfolge der Häufigkeit :

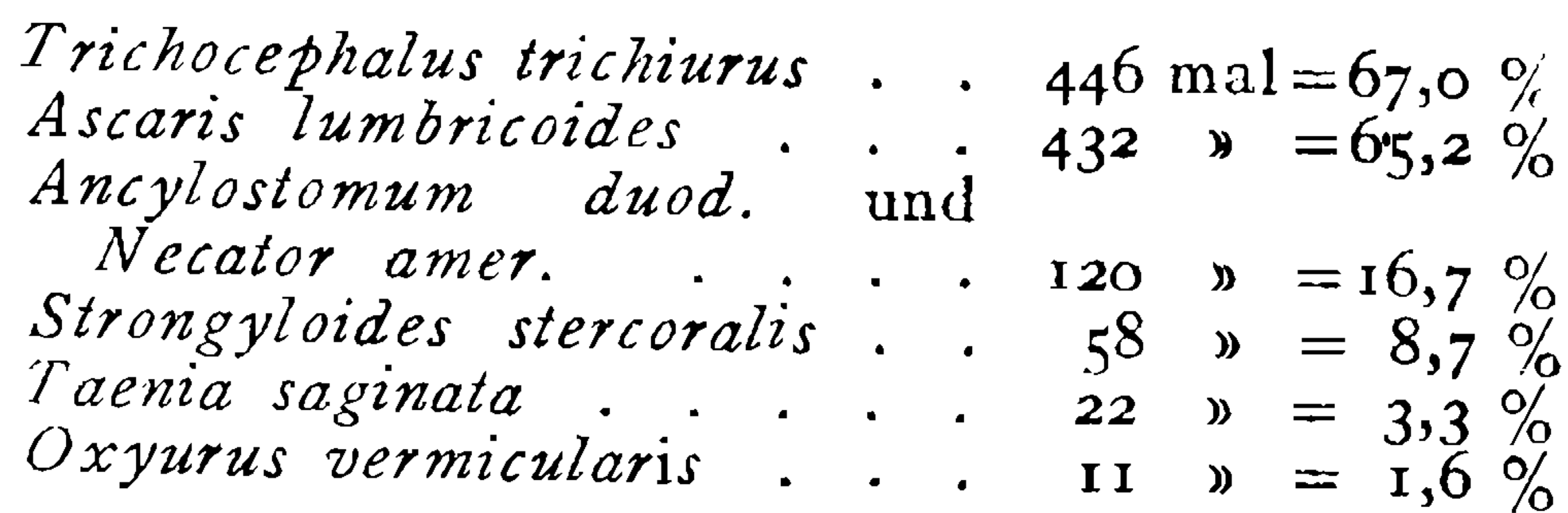

In Betreff der Häufigkeit der Parasitenassoziationen gebe ich folgende Zahlen :

Ascaris und Trichocephalus . . . . $234 \mathrm{mal}$ Ascaris, Trichocephalus und Ancy. lostomum . . . . . . . . . 4 I Ancylostomum und Trichocephalus: 22 》 Ancylostomum, Trichocephalus und Strongyloides . . . . . . . . 20 》 Ascaris und Ancylostomum . . . . $\quad$ I5

Ascaris, Trichocephalus, Ancylostomum und Strongyloides . . . . I2 》 Trichocephalus und Strongyloides : 7 " Ascaris und Strongyloides. . . . 7 " Ascaris, Trichocephalus und Strongy-

loides . . . . . . . .

stomum und Strongyloides. 2 .

Trichocephrlus und Oxyurus. . . I n

Ascaris, Trichocephalus und Taenia I D

Ascaris, Trichocephalus, Ancylosto. mum und Oxyurus.... . .

Bloss durch einen Parasiten hervorgerufene Infektionen beobachtete ich in nachfolgenden Verhältniszahlen :

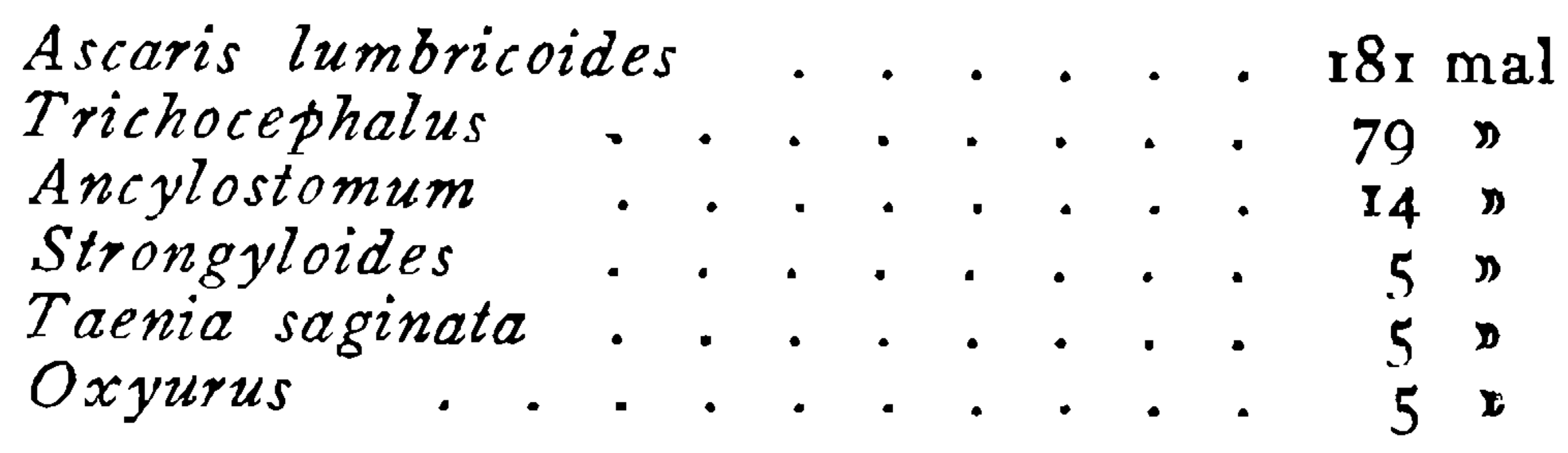

Um die Häufigkeit der Parasiten in den verschiedenen Altersklassen zu zeigen, stellte ich folgende Tabelle auf, welche die Prozentzahlen für jedes Geschlecht getrennt wiedergibt : 


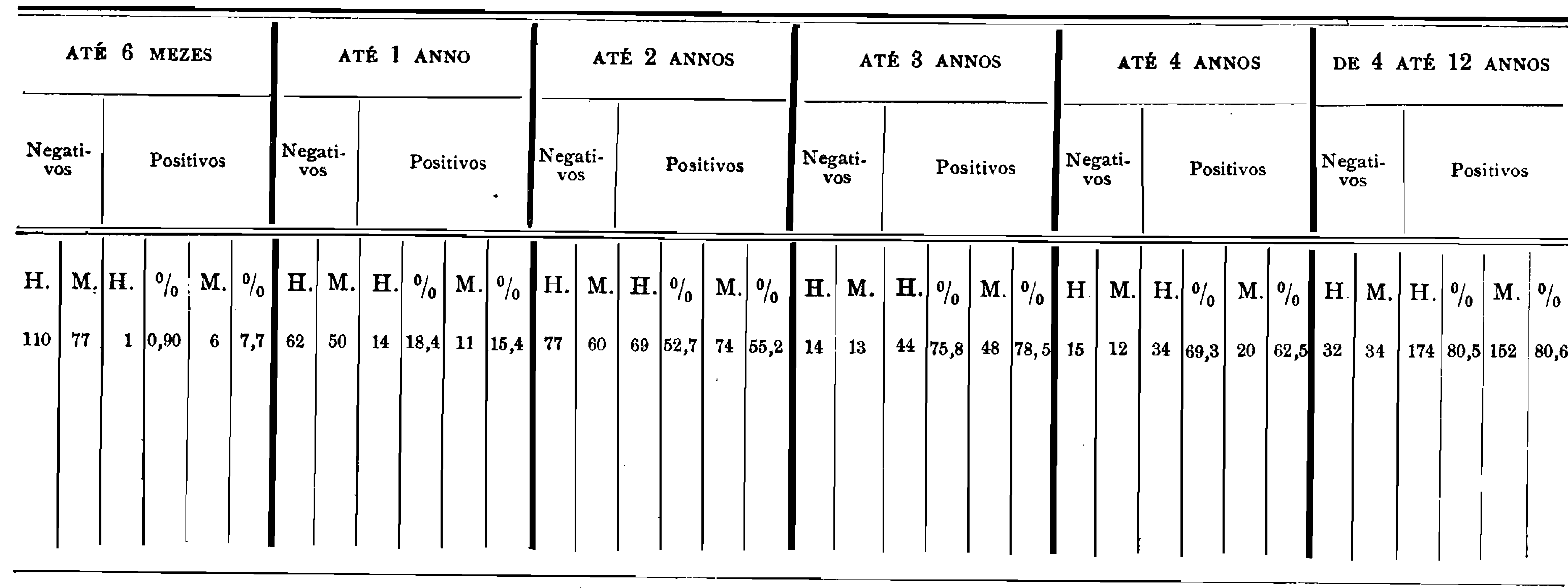

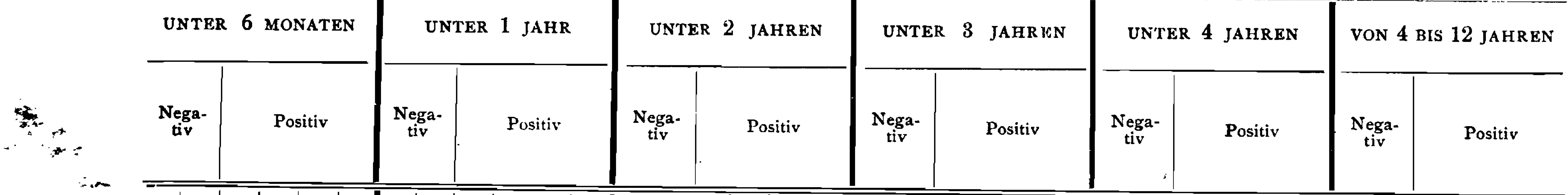

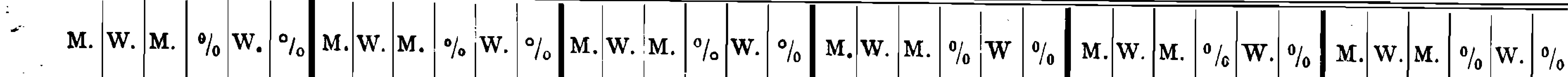

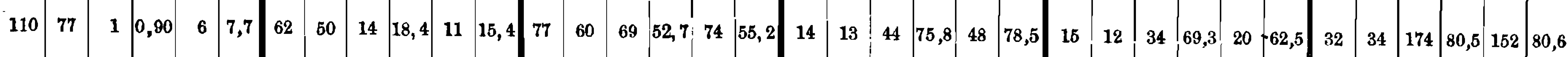


Aproveitamos o ensejo para dar algumas notas interessantes acerca dos parazitos intestinaes do homem.

As duas especies de ancilostomo que parazitam o homem foram por nós encontradas apoz a administração dos antielminticos conhecidos e uzuais, não nos sendo possivel estabelecer uma percentajem, visto que o tratamento não pode ser feito em todos os cazos ; comtudo o Necator americanus Stiles reprezenta, entre nós, a especie predominante como já verificaram outros observadores. Todas as tenias, por nós observadas, pertenciam á especie Taenia saginata L. Atenção muito especial foi consagrada á pesquiza das outras especies, principalmente das do genero Hymenelopis e do Dipylidium caninum L, que varios autores têm constatado serem de não rara frequencia na infancia, apezar disso nenhum cazo nos foi dado observar.

É para notar a auzencia de infestações com Taenia solium que consideramos ser bastante rara entre nós, visto que em grande numero de cazos de solitaria que temos observado, mesmo em adultos, sempre o parazito se refere a Taenia saginata.

O mesmo fato observou Stiles (I895) para a America do Norte contradizendo a RAILliET em uma nota sobre a raridade da Taenia solium. TANAKA (I9I0) em um estudo sobre os parazitos intestinais do Japão nos informa da absoluta auzencia aí da Taenia solium.

É para notar que na inspeção das carnes de nossos matadouros ocorre com frequencia a forma larvaria desta Taenia ou Cysticercus cellulosae nos musculos dos porcos ; tambem a literatura medica nacional rejista alguns cazos de cisticercoze humana.

É possivel que no interior do paiz, onde o uzo da carne de porco é feito em maior escala que nas cidades, o mesmo não aconteça, mas nesse ponto ha carencia absoluta de dados seguros. Quanto á frequencia de Taenia saginata em nossa estatistica, é possivel que seja um pouco exagerada, pois em alguns cazos a prezença de sintomas suspeitos foi cauza do exame.
Ich benütze die Gelegenheit zur Mitteilung einiger interessanter Notizen über die menschlichen Darmparasiten.

Die zwei Ancylostomumarten, die beim Menschen vorkommen, wurden von mir nach Darreichung der bekannten und gebräuchlichen Antihelminthica gefunden ; ich kann aber keine Prozentzahl aufstellen, weil die Behandlung nicht in allen Fällen gemacht werden konnte. Jedenfalls ist hier zu Lande der Necator americanus StILEs die häufigere Art, wie schon andere Beobachter gefunden haben. Alle Tänien, die ich beobachtete, gehörten zu Taenia saginata L. Ich schenkte auch dem Vorkommen anderer Bandwürmer, besonders Hymenolepisarten und Dipylidium caninum L. besondere Aufmerksamkeit, da diese von verschiedenen Autoren bei Kindern nicht selten gefunden wurden; trotzdem gelang es mir nicht, einen Fall aufzufinden.

Das Fehlen von Infektionen mit Taenia solium ist bemerkenswert; ich halte sie hier zu Lande für selten, da unter zahlreichen Bandwurmfällen, welche ich auch bei Erwachsenen beobachtete, der Parasit immer zu Taenia saginata gehörte.

Stiles gab 1895 dasselbe für Nordamerika an und widersprach RAILLIET in einer Notiz über die Seltenheit der Taenia solium. In einer Untersuchung über die Darmparasiten in Japan berichtet TANAKA (г9ro) das vollständige Fehlen der Taenia solium.

Es ist bemerkenswert, dass bei der Fleischschau in unseren Schlachthofen die Finne dieses Bandwurms, der Cysticercus cellulosae, im Fleische der Schweine häufig gefunden wird und auch die einheimische Fachlitteratur einige Fälle menschlicher Finnenerkrankung anführt. Es ist möglich, dass im Innern des Landes, wo mehr Schweinefleisch konsumiert wird, als in den Städten, die Sache anders liegt, doch fehlt es in dieser Hinsicht an sicheren Angaben. Die Häufigkeit der Taenia solium in meinen Angaben ist vielleicht etwas übertrieben, da manchmal das Bestehen von Symptomen zur Untersuchung führte. Ich muss noch anführen, dass auch 
Devemos ainda fazer notar que atenção especial tambem mereceu a pesquiza de ovos de Schistosomum (encontrados no Estado da Bahia por varios observadores), sendo para nós infrutifera.

Para terminar chamaremos a atenção para a idade minima, em que observámos 0 parazitismo intestinal $\mathrm{e}$ isto tanto mais se justifica pela carencia de dados na literatura. Observámos uma criança de trez mezes parazitada por Ascaris, Trichocephalus e Necator e outra de 4 mezes nas mesmas condições. Num $3 .^{\circ}$ cazo de seis mezes encontrámos Ascaris, Trichocephalus e larvas de Strongyloides.

Manguinhos, Outubro de rgir.
Schistosomumeier, die in BAHIA von mehreren Beobachtern gefunden wurden, mit besonderer Aufmerksamkeit gesucht, aber stets vermisst wurden.

Schliesslich möchte ich noch besonders auf das niedrigste Alter hinweisen, in welchem ich Darmparasiten fand und dies um so mehr, als in der Litteratur diesbezügliche Daten fehlen. Ich fand ein dreimonatliches Kind, welches Ascaris, Trichocephalus und Necator beherbergte und ein anderes von 4 Monaten, welches im selben Falle war. Bei einem dritten Patienten von sechs Monaten fand ich Ascaris, Trichocephalus und Strongyloideslarven.

Manguinhos, Oktober I9Ir.

\section{BIBLIOGRAFIA.}

Bretwer, ISAAC W. . . 1910

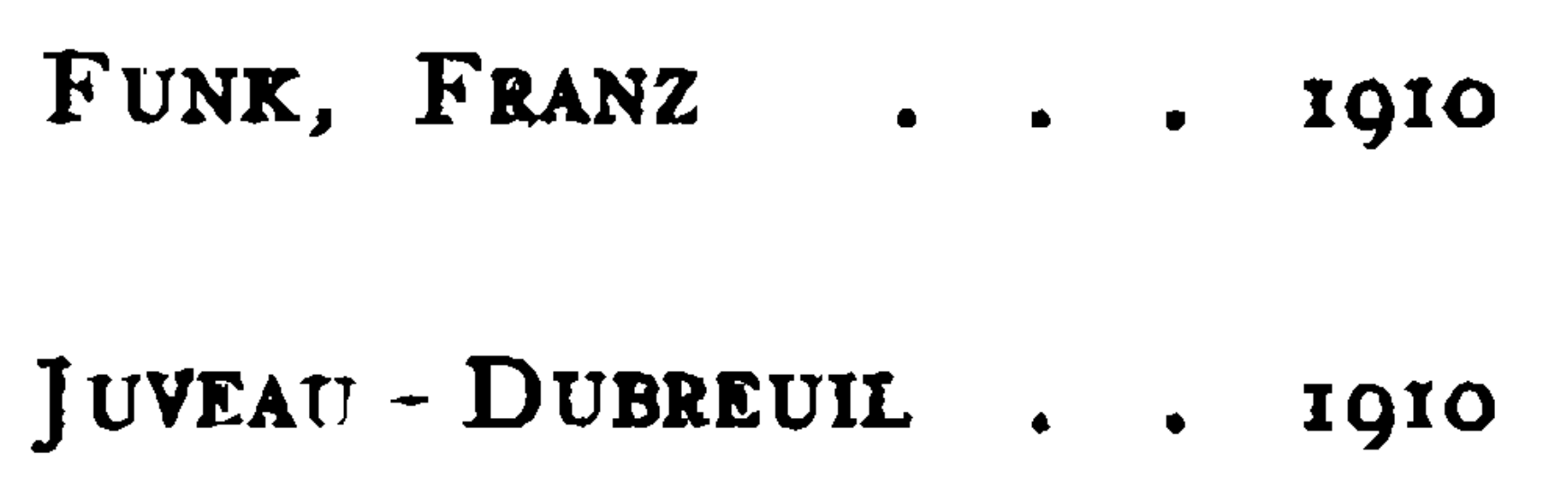

I.EICHTENSTER OTTON . IgOS

Martins, Pedro . . 1909

Mathis, C. \& Leger, M. 1909

Mathis, C. \& Leger . tgro

MIURA, M. \&

NishiUchi, N. . . IgO2

Piraja, Cesar . . . igog

SANT'ANNA, F. . . . IgIo

Sarabia y Pardo, J. . IgIo

Schloss, 0.

Schloss, Oscar
The animal parasites found in the intestines of native children in the Philippine Islands. N.Y. Med. Journ. May.

Ueber die Verbreitung von Trichocephalus dispar und anderen Helminthen-Arten.

Med.-Naturwiss. Archiv Bd. II, Heft, III. April t.

Présence de l'Oxyure vermiculaire au Tonkin.

Bull. de la Soc. méd. chirurg. de l'Indochine. Tome 1. N. 9 .

Studien über Strongyloides stercoralis (BAVAY).

Arbeit. a. d. Ksl. Gesundheitsamte, pg. 309.

Ovo helminthoscopia clinica.

Dissertação inaugural. Bahia.

Helminthiase intestinale et hépatique chez les indigènes du Tonkin et du Nord-Annam.

Bull. Soc. Path. exotique, pg. 488.

Helminthiase intestinale chez les Européens au Tonkin.

Bull, de la Soc. méd. cirurg. de l'Indochine. Tome I. N. ro.

Ueber befruchtete und unbefruchtete Ascarideneier im mensch. lichen Kote.

Centralbl. f. Bakt \&c. Orig. Bb. 32, pg. 637 .

Schistosomiases.

Dissertação inaugural. Bahia.

Estudos de helminthologia.

Acrhivos de Hygiene e Pathologia exotica. Vol. II. Fasc. I.

Solitaria em criança de ro mezes.

Brazil Medico. N. 32 de 22 de Agosto.

Helminthiasis in children.

Amer. Journ. of the med. Sc. N. $45^{8}$ v. 139. N. 5 .

The Dwarf-Tapeworm as intestinal parasite of children.

Archives of Pediatrics. Vol. XXVII. N. 2. 
Stcherback, A. . . Igro Contribution à l'étude du rôle pathologique du trichocéphale.

Stiles, Ch. Wardell . 1903 Report upon de Médecine. N. 8, pg. 643. hookworm distribution of Hyg. Laboratory. Treasury Department Public. Health

Stiles, Ch. Wardell \& and Marine Hospital Service. Bull. N. Io.

Garrison, PH. E. . Igo6 A statistical study of the prevalence of intestinal worms in man. Bull. 28. U.S. Publ. Health and Mar. Hosp. Serv.

Tanaka, Y. ... . . Igro Ueber die Arten der durch die tierischen Parasiten hervorgeruf. flenen Krankheiten in Japan. Münch. med. Wochenschrift. N. 49.

Telamann, Walter - 1908 Eine Methode zur Er?eichterung der Auffindung von Parasiten. eiern in den Fäces.

Deutsche med. Wochenschrift. N. 34 .

TORIOGI T. . . . . tgro The examination of faeces for eggs of intestinal parasites.

Torres, O. . . . Igog Contribuição ao estudo dos ankylostomos na Bahia.

Wells, C. A. . . . ro The Uncinaria de doutoramento. Bahia.

Journal of the Amer. Med. Association. Vol. Liv. 23. 